\title{
Tratamiento endovascular de 473 aneurismas intracraneanos: resultados angiográficos y clínicos Experiencia colombiana, 1996-2008
}

\author{
Sergio Alberto Vargas ${ }^{1,2}$, Diego Alberto Herrera ${ }^{1,2}$, José William Cornejo 3 \\ 1 Departamento de Radiología, Universidad de Antioquia, Medellín, Colombia \\ 2 Departamento de Neurorradiología, Centro Avanzado de Diagnóstico Médico, CediMed, Medellín, Colombia \\ ${ }^{3}$ Departamento de Neuropediatría, Universidad de Antioquia, Medellín, Colombia
}

Introducción. La terapia endovascular es una técnica aceptada mundialmente para el tratamiento de aneurismas cerebrales rotos y no rotos. En nuestro medio no hay grandes series publicadas que permitan comprobar la reproducibilidad del método según el grado de oclusión y la morbimortalidad de la técnica.

Objetivo. Evaluar los resultados angiográficos y clínicos de la oclusión endovascular de 473 aneurismas intracraneanos tratados durante un período de 12 años.

Materiales y métodos. Se realizó un estudio retrospectivo en 376 pacientes con 473 aneurismas intracraneanos tratados por terapia endovascular entre los años 1996 y 2008. Se analizaron las imágenes angiográficas antes del procedimiento y después de él y se registró el estado clínico al momento de ser dado de alta, según la escala de coma de Glasgow. Se evalúo la morbimortalidad asociada con la técnica y los resultados angiográficos inmediatos.

Resultados. La factibilidad técnica del procedimiento fue de 96,8\%. Según la escala de Hunt y Hess, al momento de la admisión, 8,7\% de los pacientes estaban en el grado I, 35,7\% en el grado II, 19,2\% en el grado III y $7,8 \%$ en los grados IV o V. El $28,5 \%$ de los pacientes tenían aneurismas no rotos. Los resultados angiográficos inmediatos demostraron oclusión total en $69,6 \%$, presencia de cuello remanente en $17,5 \%$ y oclusión parcial o fallida en $16,1 \%$ de los pacientes con "embolización". La mortalidad global fue de $10,4 \%$, con una mortalidad del procedimiento de $1,5 \%$. Se presentaron complicaciones asociadas con la técnica en 7,6\% de los aneurismas tratados.

Conclusión. El tratamiento endovascular de los aneurismas intracraneanos se asoció con una baja morbimortalidad y una gran factibilidad técnica. Los resultados fueron similares a diversas series publicadas en la literatura mundial, respaldando la reproducibilidad del método en nuestro medio.

Palabras clave: aneurisma intracraneal, hemorragia subaracnoidea, embolización terapéutica, indicadores de morbimortalidad, angiografía cerebral, hemorragias intracraneales.

\section{Endovascular coil occlusion of 473 intracranial aneurysms: angiographic and clinical results}

Introduction. Endovascular therapy is a technique accepted throughout the world for the treatment of ruptured and non-ruptured intracranial aneurysms. In Colombia, however, no summary data have been published that validate the method in terms of occlusion grade and morbimortality.

Objective. The clinical and angiographic outcomes were evaluated for endovascular embolization of 473 intracranial aneurysms treated during a 12 year time interval.

Materials and methods. Between 1996-2008, 473 intracranial aneurysms treated by an endovascular approach were included in the current retrospective study. Pre and postprocedural angiographic images were analyzed, and clinical outcome was recorded according to the Glasgow coma scale. Immediate angiographic results and morbimortality were evaluated.

Results. The technical feasibility of the procedure was $96.8 \%$. According to Hunt and Hess scale, $8.7 \%$ patients were classified as grade I, 35.7\% grade II, 19.2\% grade III and 7.8\% grades IV/V. Non-ruptured aneurysms had occurred in $28.5 \%$ of patients. Immediate procedural angiography demonstrated total occlusion in $69.6 \%$, neck remnant in $17.5 \%$ and partial or failed occlusion in $16.1 \%$. Global mortality was $10.4 \%$ and procedural mortality was $1.5 \%$. Technique-associated complications presented in $7.6 \%$ of treated aneurysms.

Conclusion. Endovascular treatment of intracranial aneurysms was associated with low morbimortality and high technical feasibility. The results are comparable with other published data summaries and supporting the use of the method in Colombia.

Key words: intracranial aneurysm, subarachnoid hemorrhage; embolization, therapeutic; indicators of morbidity and mortality, cerebral angiography, intracranial hemorrhages. 
La hemorragia subaracnoidea espontánea es un trastornofrecuenteconunaelevadamorbimortalidad e incidencia anual estimada en 10 casos por 100.000 habitantes; la ruptura de un aneurisma intracraneano es la responsable de $70 \%$ a $80 \%$ de los casos (1-3). La presentación de complicaciones precoces, fundamentalmente la reaparición de hemorragia, conlleva un empeoramiento del pronóstico (1).

Con la intención de minimizar ese desenlace, la tendencia actual es iniciar el tratamiento precozmente. La opción terapéutica se indica en función del estado clínico y neurológico del paciente, y de la localización y las características morfológicas del aneurisma. En este mismo contexto, la detección cada vez más habitual entre la población general de aneurismas no rotos en pacientes intactos neurológicamente, obliga a seleccionar adecuadamente entre las diferentes opciones de tratamiento disponibles en nuestro medio, como la terapia endovascular o la cirugía.

Tradicionalmente, la cirugía había sido considerada como el tratamiento ideal de los aneurismas cerebrales. Sin embargo, las primeras series clínicas con terapia intravascular ofrecieron resultados satisfactorios en pacientes de alto riesgo para la cirugía, como los ancianos con hemorragias graves y, en especial, con aneurismas rotos de la circulación posterior (3).

Ante la aparición de una modalidad terapéutica alternativa menos invasiva como la terapia endovascular, durante los últimos 15 años, se ha estudiado la factibilidad de la técnica y se han publicado en la literatura mundial los resultados clínicos en múltiples centros (4-24). Además, los resultados del International Subarachnoid Hemorrhage Trial demostraron la superioridad clínica del tratamiento intravascular comparado con la cirugía abierta en aneurismas rotos $(25,26)$. Ante la aceptación mundial de los beneficios de la terapia endovascular para el tratamiento de los aneurismas cerebrales, se hace necesario comprobar la reproducibilidad del método en nuestro medio, según el grado de oclusión y la morbimortalidad de la técnica.

\section{Correspondencia:}

Diego Alberto Herrera, Calle 32D № 80B-34, Medellín, Colombia

Teléfono: (574) 412 8033; fax: (574) 3112018

herrera.diego@gmail.com

Recibido: 29/12/09; aceptado:06/08/10
El objetivo de este estudio fue evaluar los resultados angiográficos inmediatos (factibilidad, grado de oclusión) y clínicos (morbimortalidad) de la terapia endovascular en Colombia, entre los años 1996 y 2008, comparándolos con otras series.

\section{Materiales y métodos}

Se analizó retrospectivamente una serie consecutiva de pacientes que cumplieran los siguientes criterios de inclusión: procedimiento realizado entre enero de 1996 y marzo del 2008, decisión de intervención tomada en junta médica, y disponibilidad de estudios angiográficos y registros clínicos.

Se trataron 473 aneurismas intracraneanos por terapia endovascular en 12 centros neurorradiológicos de Colombia, la mayoría de ellos en Medellín. En las terapias endovasculares realizadas, siempre participó un neurorradiólogo con entrenamiento en neurointervencionismo. El abordaje intravascular para todos los casos en este estudio fue similar en cuanto a técnica, manejo hospitalario y seguimiento.

\section{Pacientes e información clínica}

La decisión de tratar al paciente con terapia endovascular se estableció por consenso entre el neurocirujano tratante y el neurorradiólogo intervencionista, considerando factores como edad, presentación clínica, localización, tamaño y forma del aneurisma.

Al paciente -cuando sus condiciones clínicas lo permitían-y a la familia se les informó sobre el procedimiento intravascular y sus riesgos. Se firmó en todos los casos un consentimiento informado.

La gravedad clínica de la hemorragia subaracnoidea se evaluó al ingreso con la escala de Hunt y Hess (27). En los pacientes que presentaban hidrocefalia, drenó líquido cefalorraquídeo antes del procedimiento intravascular.

Durante el procedimiento, con base en una referencia externa, se calculaba el tamaño del saco aneurismático teniendo en cuenta la corrección por aumento. Para la evaluación retrospectiva del tamaño del aneurisma (en las placas impresas en acetato), no se disponía en todos los casos de la medida exacta calculada por el método antes mencionado, por lo cual fue necesario basarse en una referencia interna, como el diámetro de la carótida interna supraclinoidea antes de la bifurcación $(4,5 \mathrm{~mm})$ comparado con el diámetro mayor del saco aneurismático (28). 


\section{Procedimiento}

La "embolización" se realizó bajo anestesia general. Se utilizaron catéteres guías de calibre 6 o 7 Fr. Se usaron microcatéteres y microguías para acceder al aneurisma. Para prevenir la formación de trombos en estos catéteres, se mantuvo un lavado de presión continua con solución salina con heparina y nimodipina durante todo el procedimiento.

\section{Embolización con catéter único y coils (espirales)}

A través de un catéter guía Envoy 6 Fr, se pasaba una microguía Transend 0,014 con punta floppy y microcatéter Excelsior 1018; se accedía al aneurisma y se producía una embolia con coils matrix 360; se realizaba un movimiento de la punta del catéter para acomodar una buena canasta que cubriera el cuello del aneurisma y luego se empaquetaba con coils ultrasoft de relleno con medidas decrecientes.

\section{Embolización asistida con stent}

La técnica para la colocación de stents intracerebrales (endoprótesis vasculares) puede diferenciarse en tres diferentes etapas, según los materiales disponibles.

Inicialmente, los dispositivos provenían del campo cardiológico. Se trataba de stents, en su mayoría, de acero inoxidable, expandibles por balón. Para su implantación era necesaria la utilización de sistemas triaxiales (catéter $8 \mathrm{Fr}$, catéter $6 \mathrm{Fr}$ y microsistemas). La técnica también demandaba la utilización de sistemas de intercambio y el empleo de microguías disponibles en el momento que, en su mayoría, eran de gran rigidez y poca recubierta hidrofílica, lo que limitaba su navegación distal en los vasos cerebrales. Para la implantación de este tipo de stents coronarios, se requería inflar el balón con el empleo de un manómetro. La gran dificultad radicaba en la navegabilidad de los sistemas, la colocación y posterior retiro del balón, lo cual podía movilizar el stent a un punto indeseado o a una liberación subóptima del mismo.

La segunda etapa la inauguró la llegada al país del primer sistema especialmente diseñado para navegación neurológica. Se trató del sistema Neuroform® (Boston Scientific). Este sistema fue concebido como un dispositivo de soporte para la utilización conjunta con los microcoils de platino. Con este tipo de sistema fue posible el tratamiento de aneurismas considerados anteriormente como intratables. El sistema puede ser liberado a través de un catéter terapéutico de 6Fr. Implica la utilización de microguías de uso neurológico de fácil navegación y, en su fase inicial, requería el empleo de microguías de intercambio para el adecuado avance de los sistemas.

El stent Neuroform ${ }^{\circledR}$ viene montado en un microcatéter de $3 \mathrm{Fr}$ de diámetro exterior y, para su liberación, se necesita un microcatéter interno de 2,4 Fr, denominado estabilizador. El stent está hecho de una aleación química de níquel y titanio denominada nitinol, que le confiere propiedades de alta flexibilidad, es autoexpandible y no requiere de un balón para su liberación.

Una vez liberado el stent en posición, con la adecuada cobertura del cuello de la lesión, se procedía a atravesar las celdas del stent con un microcatéter de 0,21 pulgadas de diámetro interno; se colocaba la punta del microcatéter dentro del aneurisma y se iniciaba la "embolización" con microcoils de platino.

Recientemente, este tipo de stent compuesto de nitinol, autoexpandible y de adecuada flexibilidad, ha sido perfeccionado. Es decir, para su implantación no se requiere de una microguía colocada distalmente $\mathrm{y}$, además, pueden ser introducidos a través de microcatéteres de 0,21 pulgadas de diámetro interno. La colocación de microcoils se ha visto igualmente facilitada con el empleo de la técnica en "encarcelado", en la cual el microcatéter portador del stent se deja en posición y, en forma coaxial, un segundo microcatéter se deja en el interior del aneurisma inmediatamente antes de la liberación del stent. Con esta técnica se evita el paso del microcatéter a través de las celdas del stent, lo que, a veces, ocasionaba movimientos no deseados del dispositivo.

\section{Sacrificio de vaso con balón}

En el caso de aneurismas gigantes, cavernosos o del sifón carotideo en los que no era posible un tratamiento reconstructivo, se aplicaba esta técnica. Con el paciente despierto se practicaba una angiografía completa con compresión carotídea externa para evaluar la circulación colateral a través del polígono de Willis. Si ésta era adecuada, se procedía a anticoagular el paciente con $5.000 \mathrm{U}$ de heparina e inflar el balón durante media hora (test de oclusión), para comprobar que no presentara ningún déficit motor ni del lenguaje. $\mathrm{Si}$ el paciente toleraba la prueba, se sacrificaba el vaso padre con un balón desprendible. Después del procedimiento, pasaba a la unidad de cuidados 
intensivos, evitando presiones arteriales medias menores de 80 y mayores de $110 \mathrm{~mm} \mathrm{Hg}$.

\section{Embolización con Onyx $\circledast$}

En los casos de aneurismas de cuello muy ancho de la carótida interna, en los que era difícil por la tortuosidad navegar un stent, se decidió utilizar esta técnica.Bajoanestesiageneralyconanticoagulación plena (sólo en aneurismas no rotos), a través de un catéter guía $7 \mathrm{Fr}$ y doble llave rotadora, se pasaba un balón al cuello del aneurisma (Hyperform) y un microcatéter compatible con Onyx 500® (Rebar) dentro del aneurisma. Se inflaba el balón durante 5 minutos y en 2 minutos se inyectaba el Onyx®, se dejaba fraguar y se desinflaba el balón. Cada 5 minutos se repetía el procedimiento hasta excluir el aneurisma. El balón remodelaba la luz de la carótida y no dejaba escapar el Onyx®.

\section{Esquema de antiagregación y anticoagulación}

En los casos de aneurismas rotos, se anticoagulaba al paciente con $5.000 \mathrm{U}$ de heparina sólo después de colocar el primer coil (donde hubiera alto riesgo de ruptura) y se continuaba con $1.000 \mathrm{U}$ por hora durante el transcurso del procedimiento. El introductor femoral se retiraba cuando el tiempo de coagulación activado era menor de 150.

Para los casos electivos que requerían stent (aneurismas no rotos), se usaba el esquema de antiagregación dual con $75 \mathrm{mg}$ de clopidogrel y 100 mg de aspirina al día, ambos por vía oral, por lo menos, durante tres días antes del procedimiento. En aquellos pocos casos de aneurismas con hemorragia aguda o casos urgentes que requerían stent, se utilizaba una dosis de carga de $600 \mathrm{mg}$ de clopidogrel, junto con medidas de protección gastrointestinal o, en su defecto, se empleaban los inhibidores de glucoproteína Ilb/Illa por vía arterial y posterior infusión venosa.

\section{Cuidados posoperatorios}

El cuidado posoperatorio incluía hospitalización en cuidados intensivos, monitorización invasiva de la presión arterial media, medidas para el control del vasoespasmo (terapia de hipervolemia, hemodilución e hipertensión con presiones arteriales medias entre 80 y $100 \mathrm{~mm}$ de $\mathrm{Hg}$ ), drenaje de la hidrocefalia cuando era necesario, corticoides, antiepilépticos y protectores gástricos según el criterio del médico tratante. Se registraban las complicaciones del procedimiento, su manejo y las consecuencias clínicas.

\section{Evaluación de los resultados clínicos y angiográficos}

Para todos los pacientes se disponía de copias en medio físico de las imágenes angiográficas, antes de la "embolización" y después de ella. Éstas eran evaluadas por dos neurorradiólogos. La oclusión se clasificaba como total, subtotal (presencia de remanente del cuello) o parcial ( opacidad del saco aneurismático). El resultado angiográfico se consideraba favorable si la oclusión era total o subtotal, y desfavorable, cuando el tratamiento era parcial o fallido. El resultado clínico al egreso se consignaba utilizando la escala Glasgow Outcome Scale, que incluye los grados V (buena recuperación, independiente), IV (moderadamente incapacitado, independiente), III (gravemente incapacitado, dependiente), II (estado vegetativo) y I (fallecido) (29).

\section{Análisis estadístico}

Las variables cualitativas y cuantitativas incluyeron: situación clínica del paciente, características del aneurisma y factores terapéuticos. Se describió la edad, el sexo, la condición clínica inicial (escala de Hunt y Hess) y la morfología del aneurisma (tamaño, localización y características del cuello). El tratamiento fue evaluado con variables que incluían la técnica utilizada, el número de coils, el uso de balón, el stent, las complicaciones técnicas y el grado de oclusión inmediato después del tratamiento (total, subtotal, parcial o fallido). El tamaño del aneurisma se clasificó como pequeño $(<10 \mathrm{~mm})$, grande $(10$ a $25 \mathrm{~mm}) \circ$ gigante $(>25$ $\mathrm{mm})$. La exploración de la asociación entre la oclusión exitosa y las variables independientes, se realizó empleando la prueba de $\chi^{2}$, considerando un valor de $p$ menor de 0,05 como estadísticamente significativo. Para el análisis se utilizaron los software SPSS, versión 16.0, y Epidat, versión. 3.1.

\section{Resultados}

\section{Características de los pacientes y aneurismas tratados}

El rango de edad de los pacientes estuvo entre los 3 y los 86 años, con una edad promedio de 51,7 años. El $74,4 \%$ de los aneurismas tratados se presentó en pacientes de sexo femenino. La presentación clínica se detalla en el cuadro 1. En la escala de Hunt y Hess, al momento de la admisión, $8,7 \%$ de los pacientes eran grado I, $35,7 \%$ grado II, $19,2 \%$ grado III y $7,8 \%$ grados IV o V (figura 1 ). El $28,5 \%$ de los pacientes tratados tenían aneurismas no rotos. 
Cuadro 1. Características clínicas y sociodemográficas de pacientes con aneurismas intracraneanos tratados por terapia endovascular, Colombia, 1996-2008 ( $\mathrm{N}=473)$.

\begin{tabular}{|c|c|c|}
\hline Característica & $\mathbf{n}$ & Porcentaje \\
\hline \multicolumn{3}{|l|}{ Sexo } \\
\hline Masculino & 121 & 25,6 \\
\hline Femenino & 352 & 74,4 \\
\hline \multicolumn{3}{|l|}{ Aneurisma por tratar } \\
\hline Único & 376 & 79,5 \\
\hline Múltiples & 76 & 16,1 \\
\hline Nuevo tratamiento & 21 & 4,4 \\
\hline \multicolumn{3}{|l|}{ Déficit neurológico } \\
\hline Motor & 102 & 21,5 \\
\hline Pares craneanos & 24 & 5,0 \\
\hline Lenguaje & 8 & 1,7 \\
\hline Visual & 11 & 2,3 \\
\hline Cognitivo & 2 & 0,4 \\
\hline Cerebeloso & 2 & 0,4 \\
\hline Sin déficit & 324 & 68,7 \\
\hline \multicolumn{3}{|l|}{ Presentación } \\
\hline Hemorragia subaracnoidea & 321 & 67,9 \\
\hline Incidental & 46 & 9,7 \\
\hline Efecto de masa & 38 & 8,0 \\
\hline Embolia/Infarto & 6 & 1,3 \\
\hline Otras & 62 & 13,1 \\
\hline \multicolumn{3}{|l|}{$\begin{array}{l}\text { Glasgow Outcome Score } \\
\text { (al ser dado de alta) }\end{array}$} \\
\hline I: muerte & 49 & 10,4 \\
\hline II: estado vegetativo & 0 & $0 \%$ \\
\hline $\begin{array}{l}\text { III: déficit neurológico grave/ } \\
\text { dependiente }\end{array}$ & 7 & 1,5 \\
\hline $\begin{array}{l}\text { IV: déficit neurológico moderado } \\
\text { /independiente }\end{array}$ & 37 & 7,8 \\
\hline V: sin déficit neurológico & 380 & 80,3 \\
\hline
\end{tabular}

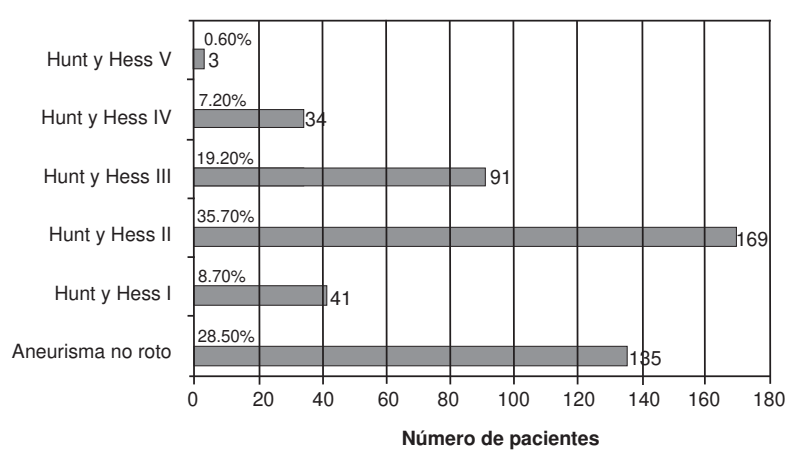

Figura 1. Clasificación según la escala de Hunt y Hess de los pacientes con aneurismas intracraneanos tratados por terapia endovascular, Colombia, 1996-2008.

La localización de los aneurismas tratados se presenta en el cuadro 2. Los aneurismas localizados en la circulación anterior representaron el 75,9\%. Los aneurismas pequeños ( $<5 \mathrm{~mm}$ ) representaron el $17,3 \%$ de la serie. Los aneurismas que midieron entre 5 y $9 \mathrm{~mm}$ fueron el $44 \%$ y, entre 10 y $25 \mathrm{~mm}$, el $36,4 \%$. Un total de 11 aneurismas $(2,3 \%)$ se consideraron gigantes (>25 mm). El $56,4 \%$ de los aneurismas tenían un cuello favorable y el $43,6 \%$ un cuello ancho (relación domo/cuello menor de 2).

\section{Tratamiento endovascular}

La mayoría de los pacientes fueron tratados mediante catéter único y "embolización" con coils (82\%). En el 10,6\% de los casos se implantaron stents. En el $6,3 \%$ fue necesario sacrificar el vaso y en 5 pacientes $(1,1 \%)$ se utilizó Onyx® con técnica de remodelado.

En 15 aneurismas el tratamiento fue fallido debido a la tortuosidad de los vasos, imposibilidad de liberar el coil o el stent en una posición adecuada o ambas condiciones. Estos pacientes fueron referidos para exclusión quirúrgica. La factibilidad del tratamiento intravascular fue de $96,8 \%$ en esta serie.

\section{Resultados angiográficos inmediatos y factores que influyeron en el grado de oclusión}

De los 473 aneurismas tratados, los incidentales correspondieron a $46(9,7 \%)$; en éstos se logró una oclusión completa en $31(64,7 \%)$, casi completa en $7(15,2 \%)$ y parcial en $6(13 \%)$. La "embolización" fue fallida en 2 (4,3\%) de los aneurismas incidentales, por dificultad para lograr el acceso por la tortuosidad de los vasos.

Al final de la intervención endovascular la oclusión se clasificó como completa en 329 aneurismas $(69,6 \%)$, subtotal en $83(17,5 \%)$ y parcial en 46 casos $(9,7 \%)$. Al clasificar el grado de oclusion según las características del cuello, encontramos que, cuando este era estrecho, el aneurisma se excluyó en $94,8 \%$ de los casos, mientras que,

Cuadro 2. Características anatómicas de aneurismas intracraneanos tratados por terapia endovascular, Colombia 1996$2008(\mathrm{~N}=473)$.

\begin{tabular}{lrr}
\hline & $\mathbf{n}$ & Porcentaje \\
\hline Localización & & \\
Carótida interna & 189 & 40,0 \\
Cerebral anterior & 88 & 18,0 \\
Cerebral media & 82 & 17,0 \\
Cerebral posterior & 10 & 2,1 \\
Eje vértebro-basilar & 104 & 22,0 \\
Tamaño (mm) & & \\
$\quad<10$ & 295 & 62,4 \\
$\quad 10$ a 25 & 153 & 32,3 \\
$\quad>25$ & 25 & 5,3 \\
Cuello & & \\
$\quad$ Estrecho/favorable & 267 & 56,4 \\
$\quad$ Ancho & 206 & 43,6 \\
\hline
\end{tabular}

Un cuello estrecho/favorable representaba una relación domo/ cuello mayor de 2.

Un cuello ancho representaba una relación domo/cuello menor de 2 . 
cuando el cuello era ancho, se logró una ocusión total en el $77,2 \%$. Al comparar las proporciones de oclusión entre estos dos grupos, se encontró que existía una diferencia significativa con un $\chi^{2}$ de 31,9 ( $p<0,000)(O R=5,34 ;$ IC95\% 2,84-10,01). Al comparar el grado de oclusión (completo o incompleto) con el tamaño del aneurisma (pequeño, grande o gigante), no se encontró diferencia significativa $\left(\chi^{2}=0,716, g \mid 2\right)(p=0,699)$.

\section{Complicaciones de la técnica intravascular}

Las complicaciones de la terapia intravascular se presentaron en 36 de los aneurismas intervenidos (7,6\%), 18 correspondientes a casos de tromboembolia (cuadro 3), presentando deterioro clínico o muerte 10 de ellos. Al discriminar la técnica empleada en las complicaciones de tromboembolia, se encontró que $88,9 \%$ de los casos fueron tratados sólo con coils, un paciente con stent $(5,55 \%)$ y en el caso restante se sacrificó el vaso.

Cuando los coágulos se visualizaban angiográficamente, se practicaba la recanalizacion mecánica con microguía y microcatéter. El tratamiento médico de esta complicación consistió en elevar la presion arterial, producir vasodilatación y fibrinólisis con un agente trombolítico (utilizando inhibidores de la glucoproteina llb/llla, pues la mayoría son trombos de plaquetas) en caso de aneurismas no rotos o en aneurismas rotos en los que se había asegurado el domo. La perforación del aneurisma o de un vaso intracraneano durante la "embolizacion", ocurrió en 12 pacientes $(2,5 \%)$, falleciendo por este motivo 5 pacientes. La mortalidad del procedimiento fue de $1,5 \%$ y la morbilidad de $2,5 \%$. La mortalidad global fue de $10,4 \%$.

De los 46 aneurismas incidentales, en 2 (4,3\%) se presentaron complicaciones. Uno fue un hombre de 42 años con aneurisma de la comunicante posterior, de $15 \mathrm{~mm}$ y cuello ancho, quien presentó perforación durante el procedimiento, deterioro neurológico y muerte. El otro caso se presentó en una mujer de 48 años, en quien se trató un aneurisma de $10 \mathrm{~mm}$ de la arteria cerebral media y quien presentó como complicación una trombosis sin consecuencia clínica. Fue tratada durante el procedimiento mediante disolución del trombo con la microguía, con restablecimiento inmediato del flujo y sin embolia distal.

\section{Resultados clínicos al momento de ser dados del alta}

En la figura 2 se presenta la mortalidad y el nivel de discapacidad según la escala de Glasgow
Cuadro 3. Complicaciones de pacientes con aneurismas intracraneanos tratados por terapia endovascular, Colombia, 1996$2008(N=473)$.

\begin{tabular}{lrc}
\hline & $\mathbf{n}$ & Porcentaje \\
\hline Complicación & & \\
$\quad$ Perforación & 12 & 2,5 \\
Tromboembolia & 18 & 3,8 \\
$\quad$ Mala posición del coil & 6 & 1,3 \\
$\quad$ Total & 36 & 7,6 \\
Consecuencias de la complicación & & \\
$\quad$ Muerte & 7 & 1,5 \\
$\quad$ Deterioro clínico & 12 & 2,5 \\
$\quad$ Ninguna & 17 & 3, \\
\hline
\end{tabular}

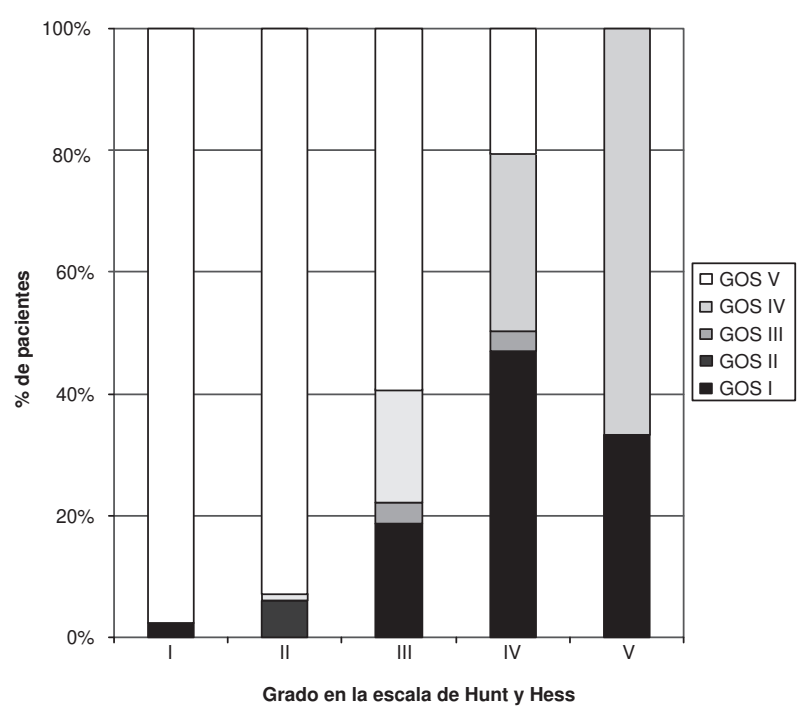

Figura 2. Relación entre la escala de Hunt y Hess al ingreso con el estado clínico al ser dado de alta de los pacientes con aneurismas intracraneanos tratados por terapia endovascular, Colombia, 1996-2008

Glasgow Outcome Score: I, muerte; II, estado vegetativo; III, déficit neurológico garve/dependiente; IV, déficit neurológico moderado/independiente; $\mathrm{V}$, sin déficit neurológico

Outcome Score, en relación con el grado clínico de la hemorragia subaracnoidea (escala de Hunt y Hess) antes del tratamiento. En cuanto al tratamiento endovascular de los aneurismas no rotos, se presentó una morbimortalidad de $9,6 \%$. En esta serie, al momento del alta $80,3 \%$ de los pacientes estaban en Glasgow Outcome Score grado V, 7,8\%; en grado IV, $1,5 \%$; en grado III, $0 \%$; en grado II, y $10,4 \%$ en grado I.

\section{Discusión}

Desde la publicación del International Subarachnoid Hemorrhage Trial, que representó el primer estudio prospectivo de asignación aleatoria, que comparó la seguridad y la eficacia de los tratamientos quirúrgico (exclusión con clip) e intravascular de aneurismas intracraneanos, se ha demostrado en 
Cuadro 4. Terapia endovascular en el tratamiento de aneurismas intracraneanos en diversas series publicadas.

\begin{tabular}{|c|c|c|c|c|c|c|}
\hline \multirow[t]{2}{*}{ Serie } & \multirow[t]{2}{*}{$\mathbf{N}$} & \multicolumn{3}{|c|}{$\begin{array}{c}\text { Grado de oclusión } \\
\text { (\%) }\end{array}$} & \multirow[t]{2}{*}{$\begin{array}{c}\text { Complicaciones } \\
(\%)\end{array}$} & \multirow{2}{*}{$\begin{array}{l}\text { Mortalidad en } \\
\text { el procedimiento } \\
(\%)\end{array}$} \\
\hline & & 100 & $>95$ & $<95$ & & \\
\hline Ascensio, et al., 2006 (3) & 71 & 50 & 42,9 & 7,1 & - & 2,8 \\
\hline Pascual-Lozano, et al., 2004 (4) & 53 & 86,8 & - & 13,2 & 9,4 & 0 \\
\hline Gruber ,et al., 1999 (11) & 31 & 68 & 13 & 19 & 13,3 & 6,7 \\
\hline $\mathrm{Ng}$, et al., $2002(15)$ & 160 & 46 & 16 & 38 & 6,9 & 1,2 \\
\hline Vanninen, et al., 1999 (17) & 52 & 55 & 38 & 6 & - & 2 \\
\hline Murayama, et al., 2003 (19) & 916 & 55 & 35,4 & 3,5 & 8,4 & 1 \\
\hline Henkes, et al., 2004 (20) & 1811 & 65,8 & 20,7 & 5,5 & 5,3 & 1,5 \\
\hline Viñuela, et al., 1997 (21) & 403 & 70,8 & 21,4 & 4,2 & 9,2 & 1,7 \\
\hline Solander, et al., 1999 (22) & 79 & 68 & - & 32 & 13 & - \\
\hline Gallas, et al., 2005 (23) & 705 & 72,6 & 25 & 2,4 & 9,5 & 1,4 \\
\hline Vargas, et al., 2008 & 473 & 69,6 & 17,5 & 12,9 & 7,6 & 1,5 \\
\hline
\end{tabular}

forma clara que el riesgo de muerte o discapacidad a 1 año luego del tratamiento se reduce de forma significativa utilizando la "embolización" con coils $(25,26)$. Las tasas de morbimortalidad son menores para la terapia intravascular que para la cirugía (26). En este contexto es importante realizar una autoevaluación de nuestros resultados, como en otras series publicadas (4-24), con el fin de establecer la reproducibilidad del método intravascular en nuestro medio.

En la presente serie, la factibilidad del tratamiento intravascular fue alta, y se presentaron solamente 15 fallas $(3,2 \%)$. Algunos de estos casos fueron tratados quirúrgicamente y otros no fueron intervenidos por sus condiciones clínicas. Esta alta tasa de factibilidad se explica por la selección inicial de los aneurismas que debían ser tratados por terapia intravascular y por la experiencia técnica de los operadores (13).

En todos los aneurismas tratados, los casos fueron discutidos y seleccionados previamente en una junta médica con la participación de neurocirujanos y neurorradiólogos, seleccionando el tratamiento más apropiado según las condiciones individuales de cada paciente. Usualmente, la terapia intravascular fue la elección inicial para aneurismas vértebro-basilares, múltiples o de circulación anterior cuando el cuello era favorable. Para los aneurismas de la arteria cerebral media, cuando el cuello era ancho se prefirió la opción quirúrgica. También, se optaba por la cirugía abierta cuando se asociaba la hemorragia subaracnoidea con un hematoma que requería drenaje.

\section{Grado de oclusión}

Se ha definido el grado de oclusión del aneurisma de forma semicuantitativa, basándose en una estimación visual subjetiva (21), aceptando que puede existir variación entre diferentes observadores. Por esta razón, el grado de oclusión se calculó por consenso entre dos neurorradiólogos, buscando reducir posibles sesgos. De cualquier modo, se ha visto que, incluso cuando hay oclusión angiográfica completa, sólo $30 \%$, aproximadamente, del saco aneurismático se encuentra lleno con el material de embolia cuando se usan coils (30-33). En este estudio se logró una oclusión total o subtotal del $87,1 \%$, acorde con lo reportado en la literatura (4-24). Lograr esta tasa de exclusión es importante, puesto que se ha visto que la oclusión parcial puede no proteger al aneurisma de las diferentes fuerzas hemodinámicas, pudiendo sobrevenir hemorragia nuevamente (21).

Sólo se encontró asociación estadísticamente significativa, entre un mayor tamaño del saco o cuello aneurismático y un menor grado de oclusión, factores críticos a la hora de excluir un aneurisma por terapia endovascular, previamente descritos por otros autores $(21,33)$. En el estudio no se encontró diferencia significativa al comparar el grado de oclusión con el tamaño del saco aneurismático, como ha sido descrito por otros (21). Este resultado probablemente se deba al bajo porcentaje de aneurismas gigantes tratados $(5,3 \%)$ y a la frecuente utilización por el autor de la técnica de sacrificio de vaso con balón en esos casos.

\section{Morbilidad y mortalidad}

El total de complicaciones fue de 7,6\%. En otros reportes publicados (4-24), diferentes autores han informado una tasa de complicaciones globales similar, entre6\% $613 \%$,y también lascomplicaciones más frecuentes fueron las tromboembolias. En esta serie no se encontró relación significativa 
entre la localización o el tamaño del aneurisma y el número de complicaciones, como fue descrito por otro autor (15); tampoco hubo influencia negativa sobre los resultados cuando se utilizaron técnicas como el remodelado o la implantación de stent.

La mortalidad del procedimiento fue de $1,5 \%$ en la presente serie. La mortalidad del procedimiento incluía las consecuencias de las complicaciones de la terapia intravascular (perforación, trombosis, embolia y mala posición del coil). En los aneurismas rotos se debe distinguir entre la evolución de la hemorragia subaracnoidea y las complicaciones derivadas del uso de dispositivos y materiales en la terapia intravascular.

Cuando existe una mala condición clínica, la muerte puede ocurrir como consecuencia del vasoespasmo - la hipertensión endocraneana (secundaria a hidrocefalia o hematoma). La mortalidad global en este estudio fue de $10,4 \%$, desenlace producto de una pésima condición clínica inicial, evidencia de vasoespasmo sintomático (evaluado clínicamente o por Doppler transcraneano) y complicaciones de la terapia intravascular. En esta serie se observó correlación entre el grado de la escala de Hunt y Hess, y la mortalidad. Sin embargo, la supervivencia de estos pacientes en malas condiciones clínicas iniciales ha mejorado con la terapia intravascular, dado que el resultado obtenido con el tratamiento quirúrgico históricamente ha sido insuficiente $(29,30)$.

Al analizar separadamente los aneurismas no rotos e incidentales de los sintomáticos, se encuentra que las tasas de ruptura a cinco años en pacientes sin historia de hemorragia subaracnoidea son de $0 \%, 2,6 \%, 14,5 \%$ y $40 \%$ para aneurismas de menos de $7 \mathrm{~mm}$, de 7 a $12 \mathrm{~mm}$, de 13 a $24 \mathrm{~mm}$ y de más de $25 \mathrm{~mm}$, respectivamente (34). Esta historia natural se debe considerar cuando se evalúan los resultados adversos después de la cirugía abierta (hasta $25 \%$ de complicaciones) o la "embolización" de aneurismas no rotos (hasta $10 \%)$, según lo reportado en la literatura (34). En la presente serie, se presentaron complicaciones en $4,3 \%$ de los casos de aneurismas incidentales. Esta información se debe valorar a la hora de comparar el riesgo de ruptura contra el riesgo de complicaciones del tratamiento electivo. En un estudio donde se calculó la expectativa de vida para determinar las circunstancias en que el tratamiento de aneurismas no rotos era benéfico, se encontró que había pérdida de años de vida en todas las edades cuando se trataron aneurismas incidentales de menos de $7 \mathrm{~mm}$ (35).

\section{Comparación de series}

Se han publicado numerosos estudios sobre los resultados angiográficos y clínicos del tratamiento intravascular de los aneurismas intracraneanos (4-24). Estos estudios han incluido pacientes con diferentes tipos de aneurismas, en diversas localizaciones, con diferentes tamaños y un estado clínico variable al ingreso, con hemorragia subaracnoidea, aneurismas no rotos o en óptimas condiciones clínicas. Para compararlos, hemos resumido los resultados de algunos de los estudios más representativos de lberoamérica $(4,5)$ y de la literatura mundial $(12,16,18,20-24)$ en el cuadro 4 , referentes a aneurismas tratados por terapia intravascular. Al evaluar todos los resultados, se apreció que la presente serie es comparable en todos los aspectos, lo que indica que el método intravascular es reproducible en nuestro medio.

En resumen, se obtuvo la oclusión completa inicial del aneurisma tratado en $69,6 \%$ de los casos, cuando lo descrito en la literatura oscila entre $46 \%$ y $86,8 \%(4,5,12,16,18,20-24)$. La morbimortalidad de la técnica en esta serie fue de $9,1 \%$, valor que se encuentra entre $2 \%$ y $20 \%$, que ha sido el rango reportado por múltiples estudios (4-24).

La realización del control angiográfico en sólo $26,8 \%$ de los casos constituye una limitación de este estudio, para obtener conclusiones acerca de la recanalización de los aneurismas intervenidos.

El tratamiento intravascular de los aneurismas intracraneanos se asocia con una baja morbimortalidad y una alta factibilidad técnica. Nuestros resultados son comparables con diversas series publicadas en la literatura mundial (4-24), lo cual respalda la reproducibilidad del método en nuestro medio.

\section{Agradecimientos}

Agradecemos a Juan Camilo Pérez, del grupo Pediaciencias, por el procesamiento estadístico de la información.

\section{Conflicto de intereses}

Ninguno de los autores declara conflicto de intereses.

\section{Financiación}

Ninguno de los autores declara fuentes de soporte financiero, vinculación con corporaciones, ni patentes involucradas. 


\section{Referencias}

1. King WA, Martin NA. Critical care of patients with subarachnoid hemorrhage. Neurosurg Clin N Am. 1994:5:767-87.

2. Guglielmi G, Vinuela F, Duckwiler G, Dion J, Lylyk P, Berenstein A, et al. Endovascular treatment of posterior circulation aneurysms by electrothrombosis using electrically detachable coils. J Neurosurg. 1992;77:515-24.

3. Tevah J. Tratamiento endovascular de los aneurismas cerebrales: sus comienzos hace 30 años y su desarrollo actual. Rev Chil Radiol. 2003;9:78-85.

4. Ascensio JL, Vargas SA, Jiménez CM, Arias SA. Terapia endovascular en aneurismas vertebrobasilares. Experiencia del grupo neurovascular. Revista Colombiana de Radiología. 2006;17:1986-94.

5. Pascual-Lozano AM, Chamarro-Lázaro R, SolerMarkessinis F, Láinez-Andrés JM. Eficacia y seguridad del tratamiento endovascular de aneurismas cerebrales: experiencia en nuestro medio. Neurología. 2004;19:36976.

6. Zubillaga A, Guglielmi G, Vinuela F, Duckwiler G. Endovascular occlusion of intracranial aneurysms with electrically detachable coils: correlation of aneurysm neck size and treatments results. AJNR Am $\mathrm{J}$ Neuroradiol. 1994;15:815-20.

7. Massoud T, Guglielmi G, Vinuela F, Duckwiler G Endovascular treatment of multiple aneurysms involving the posterior intracranial circulation. AJNR Am J Neuroradiol. 1996;17:549-54.

8. Cognard C, Pierot L, Boulin A, Weill A, Tovi M, Castaings L, et al. Intracranial aneurysms: endovascular treatment with mechanical detachable spirals in 60 aneurysms. Radiology. 1997;202:783-92.

9. Pierot L, Boulin A, Castaing L, Rey A, Moret J. Selective occlusion of the basilar artery aneurysms using controlled detachable coils: report of 35 cases. Neurosurgery. 1996;38:948-54.

10. Cognard C, Weill A, Castaings L, Rey A, Moret J. Intracranial berry aneurysms: angiographic and clinical results after endovascular treatment. Radiology. 1998;206:499-510.

11. Cognard C, Weill A, Spelle L, Piotin M, Castaings L, Rey A, et al. Long-term angiographic follow-up of 169 intracranial berry aneurysms occluded with detachable coils. Radiology. 1999;212:348-56.

12. Gruber A, Killer M, Bavinski G, Richling B. Clinical and angiographic results of endovascular coiling treatment of giant and very large intracranial aneurysms: a 7-year, singlecenter experience. Neurosurgery. 1999;45:793-803.

13. Malisch TW, Guglielmi G, Vinuela F, Duckwiler G, Gobin YP, Martin NA, et al. Intracranial aneurysms treated with Guglielmi detachable coil: midterm clinical results in a consecutive series of 100 patients. J Neurosurg. 1997;87:176-83.

14. Raymond J, Guilbert F, Weill A, Georganos SA, Juravsky L, Lambert AM, et al. Long-term angiographic recurrences after selective endovascular treatment of aneurysms with detachable coils. Stroke. 2003;34:1398-403.
15. Friedman JA, Douglas AN, Meyer FB. Guglielmi detachable coil treatment of ruptured saccular cerebral aneurysms: retrospective review of a 10-year single-center experience. AJNR Am J Neuroradiol. 2003;24:526-33.

16. Ng P, Khangure MS, Phatouros CC, Bynevelt M, ApSimon H, McAuliffe W. Endovascular treatment of intracranial aneurysms with Guglielmi detachable coils: analysis of midterm angiographic and clinical outcomes. Stroke. 2002;33:210-7.

17. Byrne JV, Sohn MJ, Molyneux AJ. Five-year experience in using coil embolization for ruptured intracranial aneurysms: outcomes and incidence of late rebleeding. J Neurosurg. 1999;90:656-63.

18. Vanninen R, Koivisto T, Saari T, Hernesniemi J, Vapalahti M. Ruptured intracranial aneurysms: acute endovascular treatment with electrolytically detachable coils: a prospective randomized study. Radiology. 1999;211:325-36.

19. Valle JN, Aymard A, Vicaut E, Reis M, Merland JJ. Endovascular treatment of basilar tip aneurysms with GDC: predictors of immediate and long-term results with multivariate analysis: 6-year experience. Radiology. 2003;226:867-79.

20. Murayama Y, Nien YL, Duckwiler G, Gobin YP, Jahan $\mathbf{R}$, Frazee $\mathbf{J}$, et al. Guglielmi detachable coil embolization of cerebral aneurysms: 11 years experience. J Neurosurg. 2003;98:959-66.

21. Henkes H, Fischer S, Weber W, Miloslavski E, Felber S, Brew S, et al. Endovascular coil occlusion of 1811 intracranial aneurysms: early angiographic and clinical results. Neurosurgery. 2004;54:268-80.

22. Vinuela F, Duckwiler G, Mawad M. Guglielmi G. Detachable coil embolization of acute intracranial aneurysm: perioperative anatomical and clinical outcome in 403 patients. J Neurosurg. 1997;86:475-82.

23. Solander S, Ulhoa A, Vinuela F, Duckwiler GR, Gobin YP, Martin NA, et al. Endovascular treatment of multiple intracranial aneurysms by using GDC. J Neurosurg. 1999;90:857-64.

24. Gallas S, Pasco A, Cottier JP, Gabrillargues J, Drouineau J, Cognard C, et al. A multicenter study of 705 ruptured intracranial aneurysms treated with Guglielmi detachable coils. AJNR Am J Neuroradiol. 2005;26:1723-31.

25. International Subarachnoid Aneurysm Trial. International Subarachnoid Aneurysm Trial (ISAT) of neurosurgical clipping versus endovascular coiling in 2143 patients with ruptured intracranial aneurysms: a randomized trial. Lancet. 2002;360:1267-74.

26. Sellar R. International Subarachnoid Aneurysm Trial (ISAT): preliminary report. Neurointerventionist. 2002;3:82.

27. Hunt W, Hess R. Surgical risk as related to time of intervention in the repair of intracranial aneurysms. J. Neurosurg. 1968;28:14-20.

28. Gabrielsen TO, Greitz T. Normal size of the internal carotid, middle cerebral and anterior cerebral arteries. Acta Radiol Diagn. 1970;10:1-10.

29. Duke BJ, Kindt GW, Breeze RE. Outcome after urgent surgery for grade IV subarachnoid hemorrhage. Surg Neurol. 1981;54:146-50. 
30. Gumprecht H, Winkler R, Gerstner W, Lumenta CB. Therapeutic management of grade IV aneurysm patients. Surg Neurol. 1997;47:54-9.

31. Kawanabe Y, Sadato A, Taki W, Hashimoto N. Endovascular occlusion of intracranial aneurysms with Guglielmi detachable coils: Correlation between coil packing density and coil compaction. Acta Neurochir (Wien). 2001;143:451-5.

32. Piotin M, Mandai S, Murphy KJ, Sugiu K, Gailloud $P$, Martin JB, et al. Dense packing of cerebral aneurysms: An in vitro study with detachable platinum coils. AJNR Am J Neuroradiol. 2000;21:757-60.
33. Hope JK, Byrne JV, Molyneux AJ. Factors influencing successful angiographic occlusion of aneurysms treated by coil embolization. AJNR Am J Neuroradiol. 1999;20:391-9.

34. van Rooij WJ, de Gast A, Sluzewski M, Nijssen PC, Beute GN. Coiling of truly incidental intracranial aneurysms. AJNR Am J Neuroradiol. 2006;27:293-6.

35. Vindlacheruvu RR, Mendelow AD, Mitchell P. Riskbenefit analysis of the treatment of unruptured intracranial aneurysms. J Neurol Neurosurg Psychiatr. 2005;76:234-9. 